\title{
Monitoring the scintillating blazar PMN J1326-5256.
}

\section{Giuseppe Cimò ${ }^{* \dagger}$}

Joint Institute for VLBI in Europe

E-mail: cimo@jive.nl

\section{Cliff Senkbail}

University of Tasmania

E-mail: cliffs@utas.edu.au

\section{Hayley Bignall}

Joint Institute for VLBI in Europe

E-mail: bignallejive.nl

\section{Simon Ellingsen}

University of Tasmania

E-mail: Simon.Ellingsen@utas.edu.au

\section{David Jauncey}

CSIRO-ATNF

E-mail: David.Jauncey@csiro.au

\section{Jim Lovell}

University of Tasmania

E-mail: jim.lovell@utas.edu.au

\section{Peter McCulloch}

University of Tasmania

E-mail: Peter.McCulloch@utas.edu.au

Scintillation of flat spectrum radio sources provides a unique instrument to study the characteristics of the electron density distribution throughout the interstellar medium as well as to study the microarcsecond structure of these compact radio objects. A multifrequency description of the intraday variability phenomenon based on the combination of Australia Telescope Compact Array observations with the data from the monitoring projects at the University of Tasmania, will be described. Some implications concerning the physics of compact structures and the characteristics of the interstellar medium will be discussed, in particular for the case of PMN J1326-5256.

Bursts, Pulses and Flickering: wide-field monitoring of the dynamic radio sky 12-15 June 2007

Kerastari, Tripolis, Greece

\footnotetext{
*Speaker.

${ }^{\dagger}$ This research has been supported by the ARC grant number DP0342500 at the University of Tasmania.
} 


\section{Introduction}

Intra-Day Variable sources (IDV) are flat spectrum extragalactic synchrotron sources, often associated with optical quasars, BL Lacs or radio galaxies, that show flux density variability on time scales shorter than a day [6]. They are typically unresolved with the longest VLBI baselines at centimetre wavelengths. These sources show intrinsic variations on much longer time scales (months) that sometimes are associated with milliarcsecond structure that evolves on time scales of years [8]. Source-intrinsic explanations of IDV require extremely compact objects that need extreme (if not impossible) values of Doppler boosting to avoid brightness temperature to violate the inverse compton limit. However, the easiest way to understand the intra-day variability is as a propagation effect, similar to that in low frequency variables, due to scintillation in an interstellar screen of ionized gas ([11] and references therein). In this paradigm, the refractive screen acts like the agitated surface of a swimming pool, that bends sunlight to cause an irregular pattern of light and dark bands on the bottom of the pool. In the astronomical case, the Earth moves through an immense pattern of light and dark bands, which causes the variability of the IDV sources.

The main characteristic of IDV is the variability on time scales of a day to less than one hour of both total $(S)$ and linearly polarized $(P)$ flux densities. The amplitudes of the fluctuations differ from source to source but are typically in the range $m_{S}=5-20 \%$, where $m_{S}$ is the total intensity flux density modulation index $\left(m_{S}=\sigma_{S} / S\right)$. Often, the variability is more pronounced in the polarized than in the total flux $\left(m_{P}>100 \%\right)$. Usually, the variations in $S$ and $P$ appear correlated but in some cases are anti-correlated [10].

An ISS origin for the IDV requires the time scales of the variations to exhibit seasonal modulations when the projected velocity of the Earth and the interstellar matter have similar speed and direction (see [11], [4]). Therefore, a proper understanding of the IDV phenomenon needs long-term continuous monitoring of IDV sources. Using the Ceduna 30-m radio telescope of the University of Tasmania, we are continuously monitoring the variability of a number of compact sources at $6.7 \mathrm{GHz}$ in order to obtain information about the variability pattern during the course of the year [9]. The Ceduna continuous monitoring program is essential to collect information about the long-term characteristics of the observed flux density fluctuations.

\section{Observations}

The remotely operated 30-m Ceduna radio telescope is located $850 \mathrm{~km}$ west of Adelaide, in South Australia. It was built by Mitsubishi in 1969, and it has been used by the Overseas Telecommunications Company (OTC) for telephone and television communication via the Intelsat satellite system up to October 1994. In September 1995, the station has been donated to the University of Tasmania. In March 2003, the COSMIC project, COntinuous Single-dish Monitoring of Intra-day variability at Ceduna (see [9]), has commenced with the aim of providing continuous observation of a sample of southern scintillators at $6.7 \mathrm{GHz}$. The long-term light curve of the BL Lac PMN J1326-5256 is shown in fig.1. The data was collected between March 2003 and November 2006 (MJD 52700 - 54060). The short gaps in the light curve are due to the observing strategy. The target sources are divided in two groups: north and south of the zenith at Ceduna. Each group 


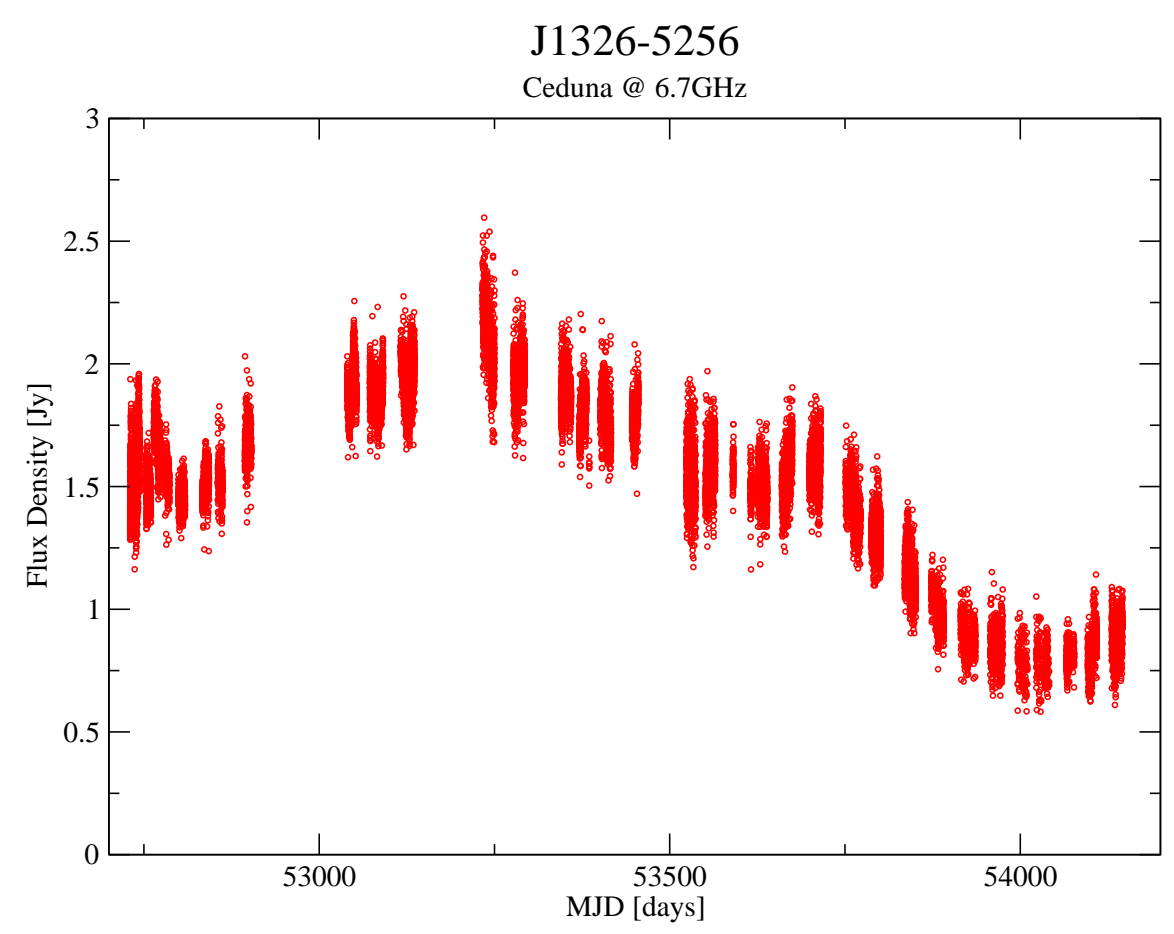

Figure 1: PMN J1326-5256 monitored at Ceduna at 6.7 GHz.

is observed for 14 days. The larger gaps are due to telescope maintenance and Australian VLBI sessions.

The time series analysis of the observations of PMN J1326-5256 over the last 3 years have shown a complex variability pattern in this intraday variable source. This object has changed its scintillation behaviour since the early observations going through the following stages: fast variations - quiescence - long-term variability - fast variations.

Here, we present observations of J1326-5256 carried out at the beginning of the COSMIC project (see fig.2), when the source suddenly changed from high variability to a quiescent phase. The total intensities of the target source and the calibrators (i.e. B1934-638) have been measured by means of cross-scans in both right ascension and declination. Each point correspond to a telescope scan in one direction. The errors on a single measurement are negligible. The data reduction and the time series analysis have been done using dedicated MATLAB procedures, written at the University of Tasmania.

Furthermore, we present data from the observations with the ATCA carried out simultaneously at 1.4, 2.4, 4.8, and $8.6 \mathrm{GHz}$ (see fig.3, total intensity on the top panels, Stokes Q and $\mathrm{U}$ on the bottom panels). The data for both total intensity and polarization have been reduced using the MIRIAD package.

The characteristic time scales are calculated from the autocorrelation function, that is the time scales on which the autocorrelation function drops to half of its maximum value. 


\section{Results}

A peculiar quiescent period has been observed in the otherwise strongly variable source PMN J1326-5256 during our Ceduna intraday variability monitoring project. We have also detected fast and pronounced polarization changes during this low-level variability phase of the total flux density using the Australia Telescope Compact Array (ATCA).

Transient IDV has been observed in a number of sources. However, the peculiar behaviour of PMN J1326-5256 differs from that seen in other episodic IDV sources. For example, in B0917+624, the amplitude of the variations changed from $\sim 10 \%$ to became "stable" at a level of 1-2\% [2], whereas in PKS 0405-385, the extreme variability lasts several months and is followed by periods of the order of the years when only slow changes are observed [7].

In the course of the Ceduna monitoring project, PMN J1326-5256 has shown pronounced IDV with a complex pattern. Our time series analysis has revealed multiple characteristic time scales: $\tau \sim 0.4$ days and $\tau \sim 1.4$ days up to long-term (>few days) variability. PMN J1326-5256 was also observed with the ATCA during monitoring of compact radio sources at 4.8 and $8.6 \mathrm{GHz}$ [1]. In this set of observations, the variations in polarized and total flux densities were strongly correlated in early 2001, but later became uncorrelated. This implies complex structure of the radio core.

Suddenly, in April 2003 (MJD 52780), the flux density fluctuations of this strongly variable object ceased on a time scale of less than two weeks (fig.2). In the following months up to December 2003 (MJD 52980), the source appeared to be stable around a mean flux density of $1.5 \mathrm{Jy}$ apart from brief episodes of variability. During these sporadic events, the flux density slightly increased and decreased with time scale of $\sim 1$ day before reverting to its quiescent phase. Measurements made in January 2004 (MJD 53020) at Ceduna show an intriguing pattern: we noticed a well-defined variability although with a longer time scale (weeks) and smaller amplitude than seen before. Furthermore, the ATCA data shows multi-frequency variability of the total flux density (in agreement with the Ceduna results), but interestingly, very fast ( $\sim 2$ hour) polarization changes have also been detected (see bottom panels on fig.3).

A possible explanation of the slow-down in the variations is that we have observed an annual modulation of the scintillation. However, such a seasonal effect can be ruled out since we know from previous observations that the source showed very strong variability in the first part of the year [1]. One could interpret this sudden change in the framework of the ISS theory as due to quenched scintillation caused by changes either in the scattering size in the ISM or in the structure of the innermost region of the source.

Variations of the electron density or change of the distance of the scattering screen could also be responsible for the cessation of the rapid variability. Alternatively, one could imagine a clumpy ISM with moving clouds at different distances. However, we need to assume that in March 2003, when we started our monitoring program, a cloud was just moving away from the line of side to explain such a fast change in the variability pattern. We therefore favour for a structural change in the radio core. In this scenario, the emission of a new jet component can lead to an apparent source size larger than the scattering size in our Galaxy, thereby canceling out the variability.

Finally, the observed complex pattern could be a mixture of scintillation (annual modulation) and source-intrinsic (i.e. pc-scale structures) effects. We have seen that the source was still scintil- 
PMN J1326-5256

calibrator: B1934-638
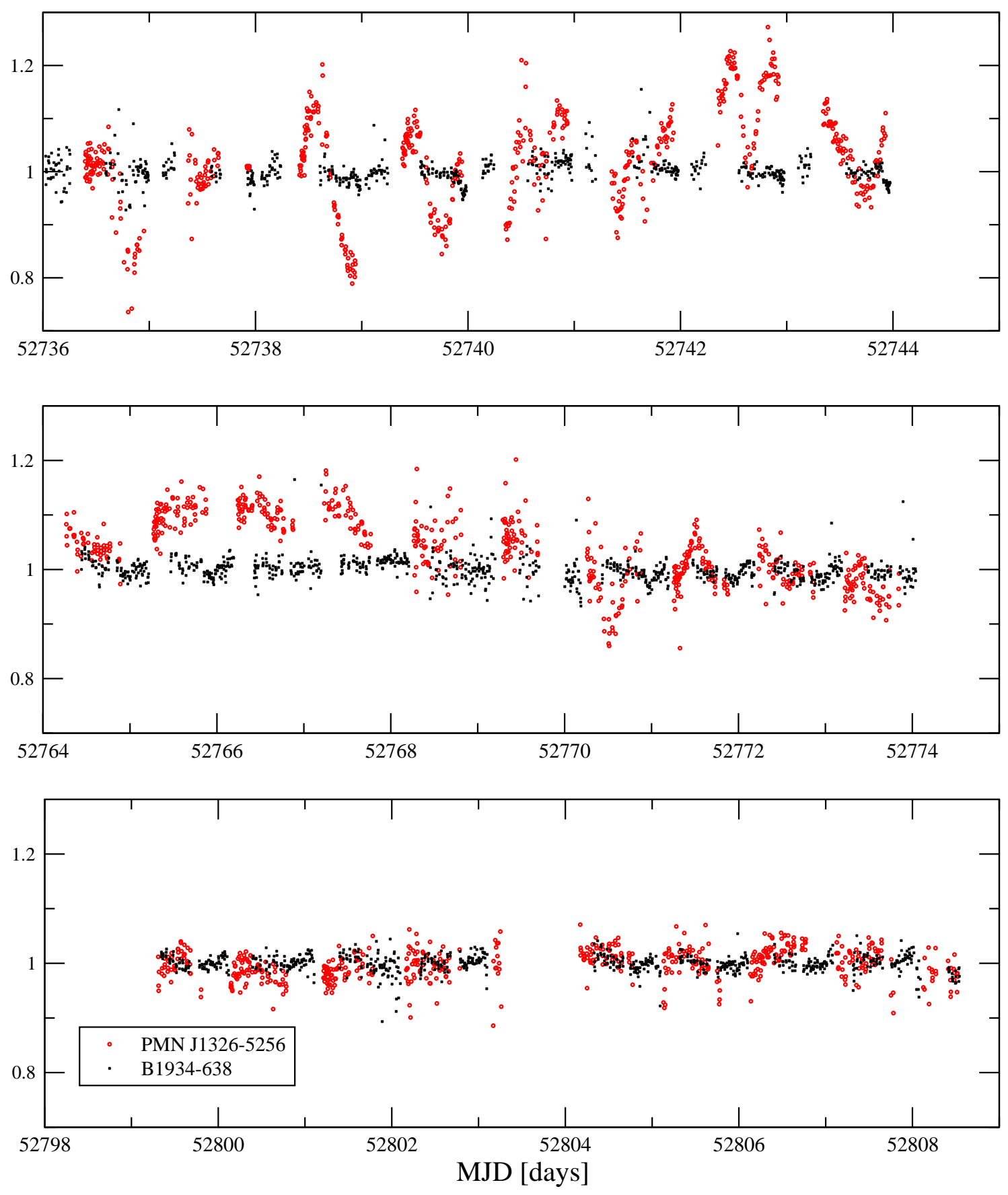

Figure 2: Intraday variations of the total flux density of PMN J1326-5256 (Red circles) at 6.7 GHz. Note the dramatic change in the nature of the temporal variability after MJD 52744. The data are normalized in order to compare the source variations to the calibrator B1934-638 (Black crosses). 

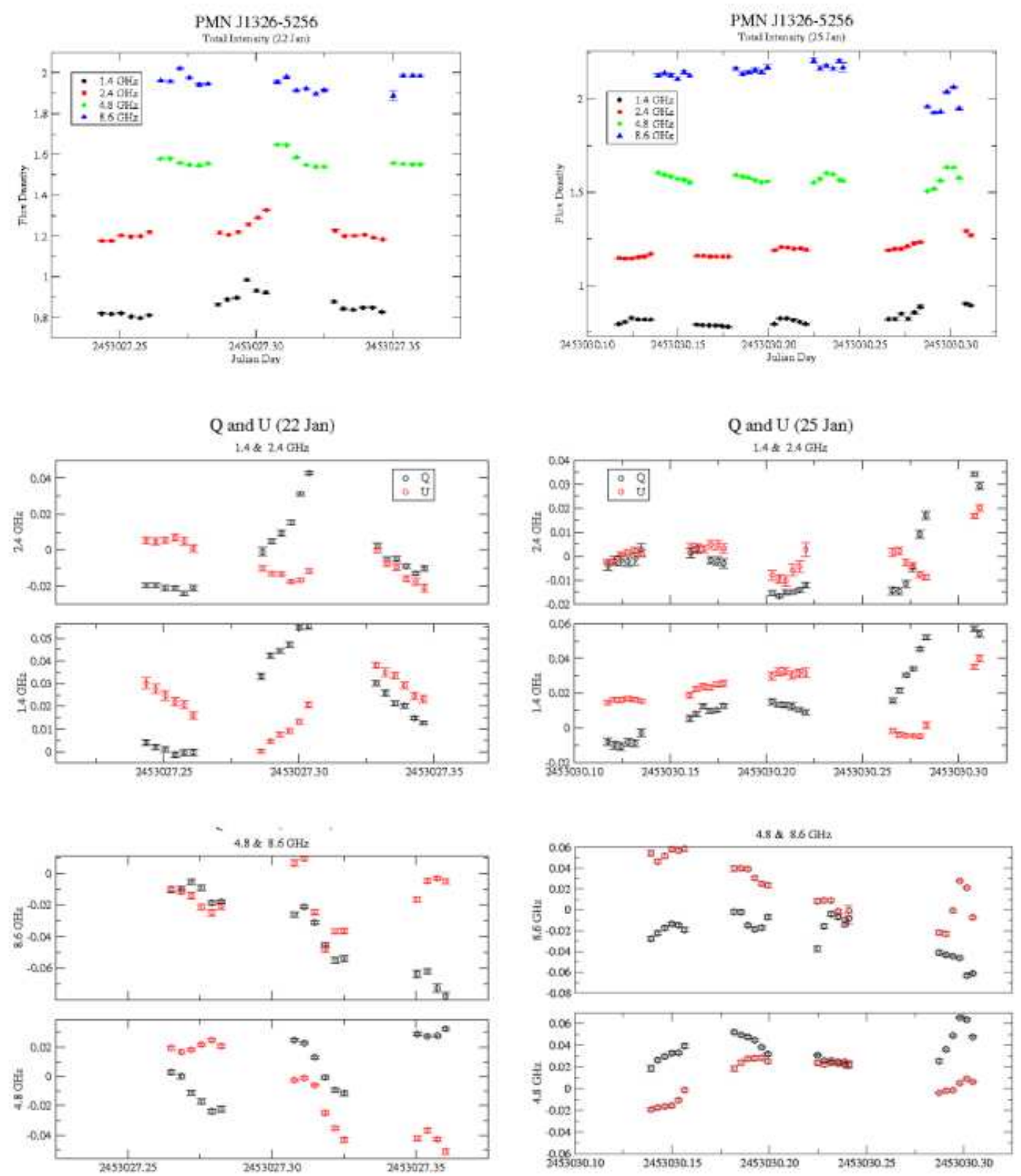

Figure 3: Intraday variations of PMN J1326-5256 at the ATCA in January 2004. Top two panels correspond to total intensity variations. Bottom four panels show $\mathrm{Q}$ and $\mathrm{U}$ variation. 
lating in the polarized components. This suggests that these structures are smaller than the scintillating total flux density component. The variability of PMN J1326-5256 is very pronounced in the polarization. The observed variations in $\mathrm{Q}$ and $\mathrm{U}$ result in rapid changes of the polarization angle. A multi-component structure of the innermost regions of the radio jet can explain the observed complex pattern. Rickett et al [12] made a fit of the polarization pattern of the extreme scintillator PKS 04054-38 using two polarized components in the $\mu$ as structure of the source.

\section{Conclusion}

Using the Ceduna 30-m radio telescope of the University of Tasmania, we monitored the variability at $6.7 \mathrm{GHz}$ of several compact sources [9] in order to establish their annual cycles and variability characteristics.

When explaining the observed sudden change in the variability pattern of J1326-5256 taking into account only propagation effects, changes in the mean properties of the ionized ISM along the line of sight should be fully responsible for the observed cessation of variability. However, one should expect also a slow-down in both Stokes Q and U, which is not the case. Considering a clumpy ISM, two screens at different distances can cause the observed sudden changes, if the edge of the nearer screen is reached at the epoch of our observations in March 2003. Alternatively, a scenario, which also takes into account the source structure at micro-arcsecond scale, seems to be more plausible in the case of PMN J1326-5256. We suggest that the source scintillation pattern has changed due to an intrinsic "long-term" change in the compact core. An observable effect of a multi-component scattered core at micro-arcsecond scale is the complex polarization scintillation pattern. Polarization variability is usually faster and stronger and can be detected also during quiescent phases of the total flux density fluctuations. This is due to the smaller sizes of the polarized components. Accordingly, we have detected fast $(\sim 2$ hour $)$ polarization changes during our ATCA experiment in late January 2004. Only the component responsible for the total intensity variations has become large enough to "quench" the scattering while we still observe the fast scintillating polarized subcomponents in the jet.

\section{References}

[1] Bignall H. E., Jauncey D. L., Kedziora-Chudczer L. L. et al. 2002, New Results from an ATCA Study of Intraday Variable Radio Sources, PASA 19, 29

[2] Fuhrmann L., Krichbaum T. P., Cimò G. et al. 2002, Annual Modulation in the Variability Properties of the IDV Source 0917+624?, PASA 19, 64

[3] Heeschen D. S. Krichbaum T. P, Schalinski C. J. \& Witzel A, 1987, Rapid variability of extragalactic radio sources, AJ 94, 1493

[4] Jauncey D. L. \& Macquart J.-P., Intra-day variability and the interstellar medium towards 0917+624, 2001, A\&A 370, L9

[5] Jauncey D. L., Bignall H. E., Lovell J. E. J. et al. 2003, Interstellar Scintillation and Radio Intra-Day Variability in "Radio Astronomy at the Fringe", Eds Zensus, Cohen \& Ros, PASP Vol 300, p 199

[6] Kedziora-Chudczer L, Jauncey D. L., Wieringa M. H. et al. 1997, PKS 0405-385: The Smallest Radio Quasar?, ApJ 490L, 9 
[7] Kedziora-Chudczer, L. 2006, Long-term monitoring of the intra-day variable quasar PKS 0405-385, MNRAS 323, 1411

[8] Krichbaum T. P., Kraus A., Fuhrmann L., Cimò G. \& Witzel, A. 2002, Intraday Variability in Northern Hemisphere Radio Sources, PASA 19, 55

[9] McCulloch P. M., Ellingsen S. P., Jauncey D. L. et al. 2005, COSMIC: Microarcsecond Resolution with a 30 Meter Radio Telescope, AJ 129, 2034

[10] Quirrenbach A., Witzel A., Kirchbaum T. P, et al. 1992, Statistics of intraday variability in extragalactic radio sources, A\&A 258, 279

[11] Rickett B. J., Witzel A., Kraus A. et al. 2001, Annual Modulation in the Intraday Variability of Quasar 0917+624 due to Interstellar Scintillation, ApJ 550L, 11

[12] Rickett B. J., Kedziora-Chudczer L. \& Jauncey D. L., 2002, Interstellar Scintillation of the Polarized Flux Density in Quasar PKS 0405-385, ApJ 5481, 103

[13] Witzel A., Heeschen D. S., Schalinski C. J \& Krichbaum T. P, 1986, Kurzzeit-Variabilität extragalaktischer Radioquellen, Mitt. Astron. Ges 65, 239 